\title{
Axonal Transport of Noradrenaline and Noradrenergic Transmission in Rats with Streptozotocin-Induced Diabetes
}

\author{
D. R. Tomlinson ${ }^{1}$, K. R. W. Gillon ${ }^{2}$ and M.G. Smith \\ Department of ${ }^{1}$ Physiology and Pharmacology, ${ }^{2}$ Biochemistry and ${ }^{3}$ Surgery (Anaesthesia), \\ The Medical School, Queen's Medical Centre, Nottingham, UK
}

Summary. The accumulation of noradrenaline in constricted sciatic nerves was measured in 6 month diabetic rats (streptozotocin $35 \mathrm{mg} / \mathrm{kg}$ ) and 4 day diabetic rats (streptozotocin $70 \mathrm{mg} / \mathrm{kg}$ ) together with two groups of age-matched control animals. There was no alteration in the amount of noradrenaline accumulated in the nerves of the diabetic animals when compared with the controls. The vasa deferentia of the long-term diabetic animals showed an impaired response to stimulation of their noradrenergic nerves and a hypersensitivity to exogenous noradrenaline. These vasa were not wasted and showed a normal contractility in response to potassium chloride. Vasa deferentia from the short-term diabetic rats showed no abnormalities of function. Vasa deferentia from all groups of rats were also examined at the ultrastructural level. Specimens from all the chronically diabetic animals contained many abnormal nerve terminals. These lesions were not seen in vasa from the shortterm diabetic rats. Taken together these findings indicate that rats with chronic streptozotocin-induced diabetes exhibit pathological changes in the noradrenergic nerves supplying the vas deferens. These animals do not, however, show an impairment of the axonal transport of noradrenaline.

Key words: Axonal transport, noradrenaline, vas deferens, sciatic nerve, diabetic neuropathies, streptozotocin diabetes.

Several groups of workers have reported that experimental diabetes caused a reduction in the axonal transport of isotopically labelled unidentified proteins and glycoproteins in various peripheral nerve trunks $[9,10,15,17,18]$. These findings, taken together with the associated decreases in conduction velocity and ultrastructural changes seen in diabetic animals [8], have prompted suggestions that abnormalities of axonal transport of structural protein and glycoprotein may be an early signal of certain forms of diabetic neuropathy $[5,11]$.

Little attention has been given to the identity of the substances which manifest reduced axonal transport, although one study demonstrated that both acetylcholinesterase and cholinacetylase activities were reduced proximal to 12 -h constrictions applied to sciatic nerves of streptozotocin-diabetic rats [16]. There exists an additional report of reduced noradrenaline accumulation in constricted sciatic nerves in diabetes, but in that case the animal was the genetically diabetic mouse [4].

The noradrenergic neurone merits attention in this context since it is known to exhibit dysfunction in many cases of diabetic autonomic neuropathy in man $[5,12,13]$. It was the object of the present study to examine the accumulation of noradrenaline proximal to a nerve constriction and to correlate the findings with the functional competence of noradrenergic transmission and the ultrastructure of noradrenergic terminals. Two groups of diabetic animals were studied; one group 3 days after induction of diabetes, the other 6 months after induction of diabetes. Streptozotocin was used in each case.

\section{Materials and Methods}

Two groups each composed of 12 male Wistar rats were made diabetic. The first group (weight range $300-330 \mathrm{~g}$; age approximately 3 months) were injected IP with streptozotocin $35 \mathrm{mg} / \mathrm{kg}$ (dissolved in $50 \mathrm{~mol} / 1$ sodium citrate buffer, $\mathrm{pH} 4.5$, immediately before injection) to produce a moderate diabetes suitable for longterm study. The second group (weight range $280-295 \mathrm{~g}$; age approximately 3 months) were given streptozotocin $70 \mathrm{mg} / \mathrm{kg}$ IP to promote a short-term diabetes of similar intensity. In each case a 
group of age-matched control animals were given a similar volume of the streptozotocin injection vehicle $(1.0 \mathrm{ml} / \mathrm{kg})$.

The animals had free access to food [Heygates $41 \mathrm{~B}$ diet (modified), L. A. Pilsbury, Birminghàm, UK] and water. Six of the diabetic rats, together with an equal number of controls were sacrificed 6 months after streptozotocin treatment. At death the blood glucose values were $36.8 \pm 2.3 \mathrm{mmol} / \mathrm{l}$ (mean \pm SEM) for the diabetic group and $8.2 \pm 0.6 \mathrm{mmol} / \mathrm{l}$ for the controls. The animals were killed by a blow on the head; both vasa deferentia were removed and transferred to ice-cold Krebs' fluid. In each case the left vas was suspended in an organ bath to examine noradrenergic transmission. A small segment from the centre of the right vas was prepared for electron microscopy.

The remaining animals of this group, together with their controls, were taken 6 months after initiation of diabetes for measurement of noradrenaline accumulation proximal to a 24 -h constriction applied to the right sciatic nerve.

The short-term diabetic animals were used in a similar fashion. Six diabetics plus six controls were killed 4 days after injection and the vasa deferentia removed. Blood glucose values were $26.7 \pm$ $1.4 \mathrm{mmol} / 1$ for the diabetics and $6.6 \pm 0.6 \mathrm{mmol} / 1$ for the controls. The remaining animals of the short-term group (six diabetics and six controls) were taken 3 days after injection and a constriction applied to the right sciatic nerve.

\section{Nerve Constriction Experiments}

The rats were anaesthetised with ether. A 1-cm incision was made in the right flank and the muscle overlying the sciatic nerve was split along the long axis of the muscle fibres. A tight ligature (prolene; 0.7 metric; Ethicon, Livingston, W. Lothian, Scotland) was placed around the sciatic nerve where it overlay the mid-point of the femur and the wound closed with Michel clips. Aseptic technique was used throughout and the duration of the anaesthesia was no longer than 5 min.

The rats were killed $24 \mathrm{~h}$ later by a blow on the head and bled from the throat. Both sciatic nerves were removed rapidly and placed on ice. The constricted nerve was cut into $3-\mathrm{mm}$ segments to give three segments proximal and two segments distal to the ligature. From the contralateral nerve two $3-\mathrm{mm}$ segments were cut for comparison with the segments immediately proximal and distal to the constriction in the ligated nerve. Each nerve segment was homogenised in $1 \mathrm{ml}$ ice-cold $3 \%$ perchloric acid. The homogenates were stored at $-80^{\circ} \mathrm{C}$ before noradrenaline assay.

\section{Noradrenaline Assay}

Nerve homogenates were centrifuged $(9,000 \times \mathrm{g}$ for $20 \mathrm{~min})$ and the supernatants retained. The pellets were resuspended in a further $0.5 \mathrm{ml} 3 \%$ perchloric acid and re-centrifuged. The two supernatants from each segment were pooled.

Catecholamines in the supernatants were assayed by high performance liquid chromatography (Nucleosil $25-\mathrm{cm}$ column, $150 \mathrm{mmol} / 1$ citrate/acetate buffer, $\mathrm{pH} 4.5$, flow rate of $1.0 \mathrm{ml} / \mathrm{min}$ ) with electrochemical detection (Anachem, Luton, UK). The method has been described elsewhere [7], though in these experiments purification of catecholamine with alumina was found to be unnecessary. Noradrenaline standards were made up in $3 \%$ perchloric acid and frozen at $-80^{\circ} \mathrm{C}$ with the nerve homogenates.

\section{Organ Bath Studies}

Vasa deferentia were suspended between parallel platinum wire electrodes in organ baths containing Krebs' fluid gassed with 95\% $\mathrm{O}_{2}, 5 \% \mathrm{CO}_{2}$. Full details of this procedure have been reported else- where [19]. The vas was connected to an isotonic transducer (Scientific and Research Instruments, Edenbridge, Kent) which was calibrated by stepwise displacement of the arm using a micrometer clamped to the transducer. The displacement to the transducer arm was linearly related to pen excursion over the full range covered by contractions of the vasa deferentia. Thus responses of all preparations are expressed in units of pen excursion.

The vasa were used to obtain frequency/response plots for field stimulation of noradrenergic nerves, concentration/effect plots for exogenous noradrenaline and responses to $\mathrm{KCl}$. The latter was used to ensure that any aberrant responses to nerve stimulation or to noradrenaline could not have arisen as a result of disorder of the smooth muscle of the vas deferens. This was necessary in view of the wasting of skeletal muscle noted in the chronically diabetic rats. To provide an additional check on this, each vas was blotted dry and weighed on removal from the organ bath. Artefactual variation in weight due to inconsistencies in the length of the portion of vas removed from the animal was minimised by working to precise anatomical co-ordinates in the dissection.

\section{Electron Microscopy}

Segments of vas deferens were placed in a pool of fixative and cut into $1-\mathrm{mm}$ cubes. The fixation procedure was that of Tranzer and Richards [2] for the ultrastructural demonstration of noradrenergic nerves. Tissue blocks were embedded in Araldite, sectioned on a Reichert OMu3 ultramicrotome and examined in a Philips EM300 electron microscope.

\section{Presentation of Results}

Results are expressed as mean \pm SEM except where otherwise stated. Comparisons of group means were made using Student's unpaired 't'test. Comparisons of linear regressions were made by conventional co-variance analysis.

\section{Results}

Both the long and short-term diabetic rats had lost weight at the time of sacrifice. The long-term group weighed $305 \pm 14 \mathrm{~g}$ whilst their controls were $438 \pm$ $10 \mathrm{~g}$. The short-term group weighed $253 \pm 8 \mathrm{~g}$ whilst their controls were $294 \pm 8 \mathrm{~g}$.

\section{Noradrenaline Accumulations}

The noradrenaline (NA) contents of the unligated nerves were consistent in both short- and long-term diabetic and in non-diabetic rats. All values lay between 3.5 and $5.0 \mathrm{pmol} \mathrm{NA} / 3 \mathrm{~mm}$ nerve. NA contents of constricted nerves were therefore not corrected for this 'background' catecholamine.

The NA accumulations for all groups of rats are shown in Figure 1. The data show that the accumulation of NA proximal to a constriction applied for $24 \mathrm{~h}$ was confined to the $3 \mathrm{~mm}$ of nerve immediately adjacent to the ligature. There was also a small accumulation of NA distal to the constriction. 


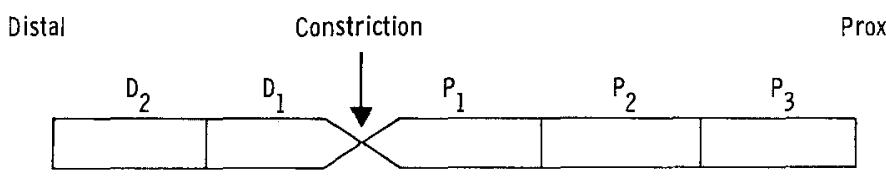

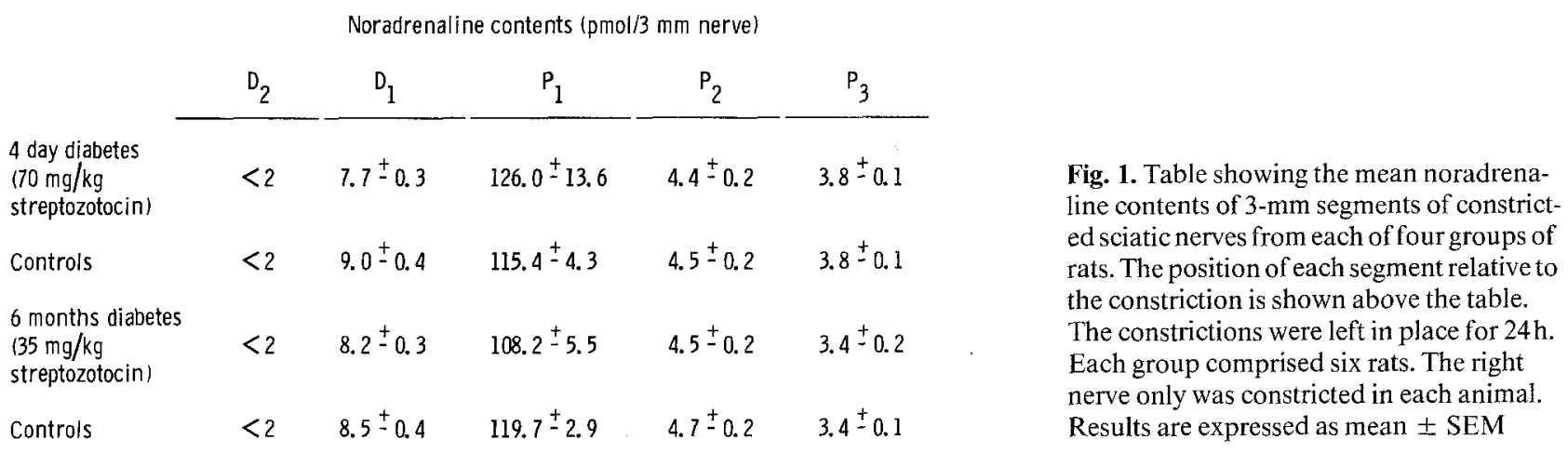

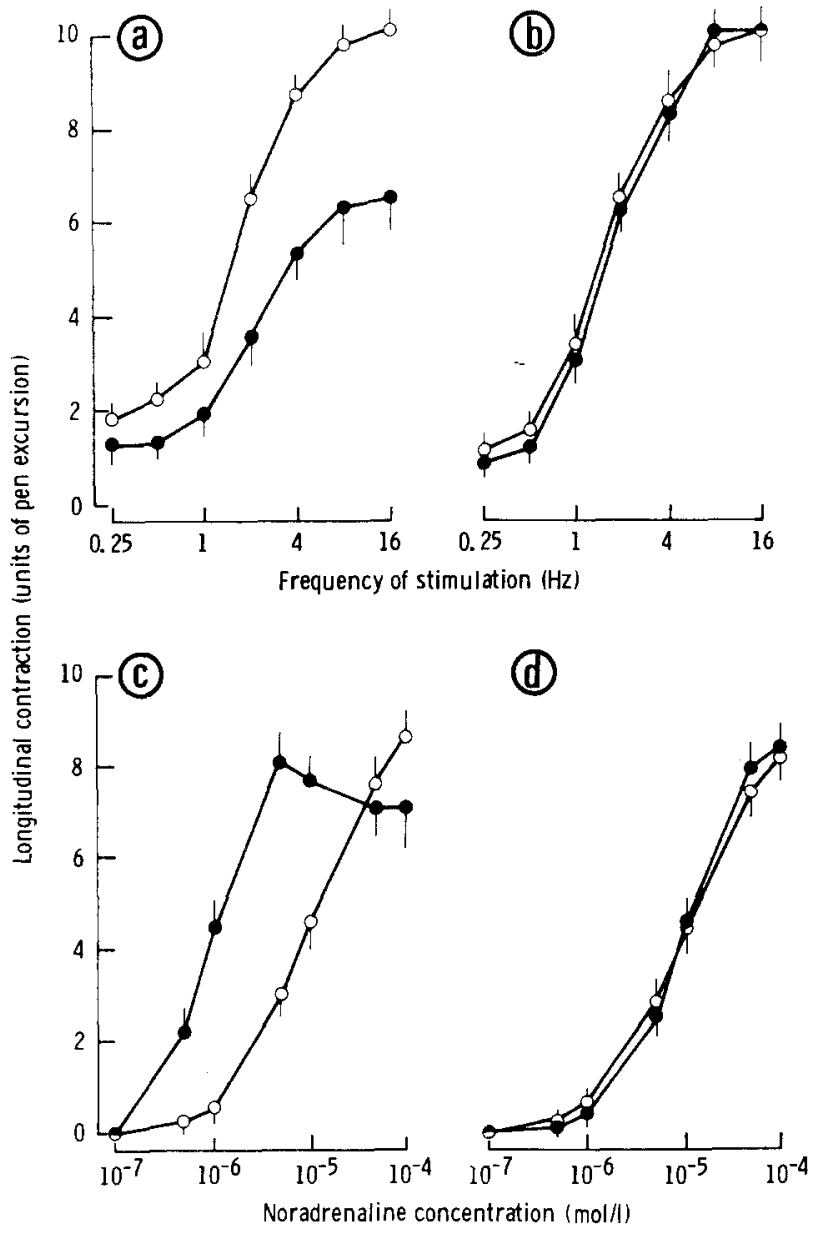

Fig. 2a-d. Responses of vasa deferentia from each four groups of rats, two diabetic ( $)$ and two control (O). (a) and (b) are frequen$\mathrm{cy} /$ response plots for field stimulation of noradrenergic nerves; (a) 6 month diabetic rats and age-matched controls, (b) 4 day diabetic rats and age-matched controls. (c) and (d) are concentration/effect plots for exogenous noradrenaline; (c) 6 month diabetic rats and controls, (d) 4 day diabetic rats and controls. Six animals comprised each of the four groups. Limit bars denote SEM
Table 1. Responses of vasa deferentia from four groups of rats to potassium chloride added to the organ bath fluid

\begin{tabular}{lll}
\hline & $\begin{array}{l}\text { Contractile response } \\
\text { to } 15 \mathrm{mmol} / 1 \mathrm{KCl} \\
\text { (units of pen } \\
\text { excursion) }\end{array}$ & $\begin{array}{l}\text { Wet weight } \\
\text { of vas } \\
\text { deferens } \\
(\mathrm{mg})\end{array}$ \\
\hline 4-day diabetic rats $(n=6)$ & $6.5 \pm 0.9$ & $72 \pm 3$ \\
Controls $(n=6)$ & $7.0 \pm 1.2$ & $76 \pm 4$ \\
6-month diabetic rats $(n=6)$ & $6.9 \pm 1.3$ & $77 \pm 6$ \\
Controls $(n=6)$ & $7.1 \pm 0.6$ & $81 \pm 4$ \\
\hline
\end{tabular}

Results are expressed as mean \pm SEM

The data show that there was no biologically significant difference between any of the groups of rats in the accumulation of NA against the ligature.

\section{Organ Bath Studies}

The response of each preparation was obtained to a dose of $\mathrm{KCl}(15 \mathrm{mmol} / \mathrm{l})$ which was found, in pilot studies, to lie on the linear portion of the concentration/effect plot. The responses to $\mathrm{KCl}$ together with the weights of the vasa deferentia are given in Table 1. The data show clearly that there were no differences in muscle contractility between preparations from the four groups of rats. The weights of the vasa were also similar indicating that no measurable wasting of this tissue had occurred in the chronically diabetic animals.

The responses of the vasa deferentia to noradrenergic nerve stimulation and to exogenous NA are shown in Figure 2. Vasa from chronically diabetic rats gave lower responses to all frequencies of stimulation than those of the age matched controls (Fig. 2a). 


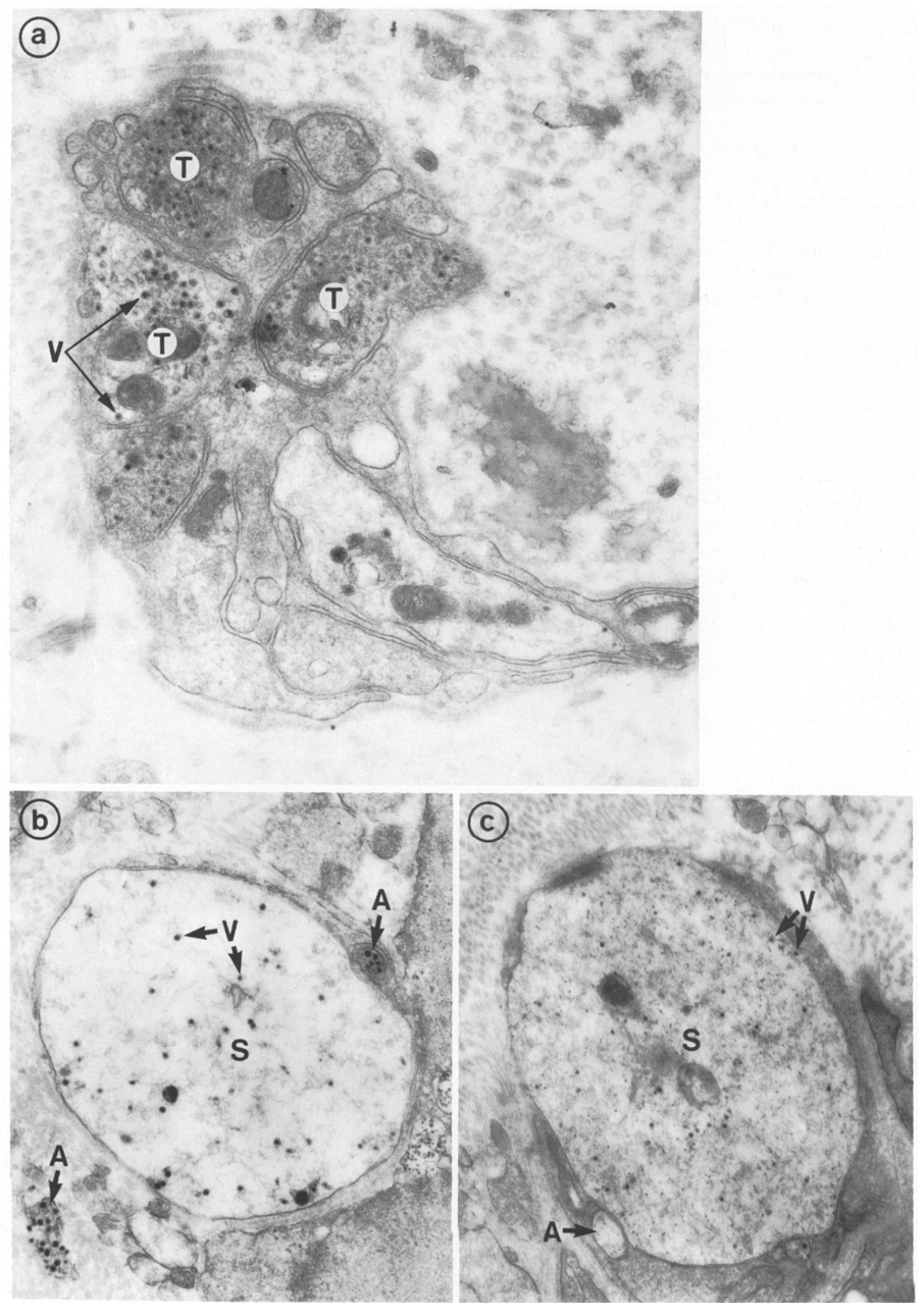

Figs. 3a-c. Electron micrographs of profiles of noradrenergic nerves from sections of vasa deferentia. (a) Control rat. Terminal $(T)$ containing many vesicles with chromaphilic cores $(V)$. (b and c) Rats with 6 month diabetes. Many terminals $(s)$ were swollen containing thinly dispersed dense-cored vesicles $(V)$ and few other axoplasmic inclusions. Many profiles of unusually small diameter $(A)$ were also present. Magnifications are (a) $38,000 \times$ (b) $29,000 \times$ (c) $20,400 \times$ 
Comparison of the linear portions of the two frequen$\mathrm{cy} /$ response plots (from 1 to $8 \mathrm{~Hz}$ ) by co-variance analysis showed that their slopes differed significantly $(p<0.05)$. There was no difference in the frequen$\mathrm{cy} /$ response plots for vasa from the acutely diabetic rats and their controls (Fig. 2b).

The vasa from the chronically diabetic rats were more sensitive to exogenous NA than were the preparations from the control animals (Fig. 2 c). Comparison of the linear portions of these two plots by covariance analysis showed that the elevations of the regression lines differed significantly $(p<0.01)$. Responses to NA of the vasa from the acutely diabetic rats did not differ from those of their controls (Fig. 2d).

\section{Electron Microscopy}

The vas deferens received a dense noradrenergic innervation. Examination of sections of vasa from both groups of control rats revealed numerous profiles of noradrenergic terminals. These were characterised by the presence of large numbers of dense cored vesicles (Fig. 3 a). Vasa from the acutely diabetic rats were indistinguishable from their controls at the ultrastructural level. In contrast, the vasa from the chronically diabetic animals differed markedly from those of control animals. About half of the nerve profiles examined in sections from all six vasa were abnormal in appearance. The most characteristically abnormal profiles were enlarged, electron lucent and almost devoid of organelles (Fig. $3 \mathrm{~b}$ ). Whether or not the apparent deficit of organelles in the swollen profiles was due to a genuine loss, or due to dilution of the normal organelle population consequent on axonal swelling, cannot be stated from the present data. Many such profiles could not be identified with certainty as degenerate noradrenergic terminals, but a large proportion contained dense-cored vesicles, making identification unequivocal. Approximately half the noradrenergic terminal profiles examined in vasa from the 6-month diabetic rats were, however, of normal appearance. Furthermore, the increase in volume of the swollen terminals would cause them to appear more frequently, as profiles in sections, than those of normal size. Thus estimates of their numbers are probably subject to systematic error.

\section{Discussion}

This study permits two main conclusions to be drawn. Firstly there was no significant reduction in the accumulation of noradrenaline proximal to a constriction applied to the sciatic nerve in rats with either moder- ate streptozotocin-induced diabetes of 6 months' duration or severe diabetes of 4 days' duration. Secondly the long-term diabetic rats showed an impaired response of the vas deferens to stimulation of its intramural noradrenergic nerves. There was no such impairment in the short-term diabetic animals.

The relation between these two findings would have been more direct if the study had been designed to measure noradrenaline accumulation in the nerves supplying the vas deferens. This was not possible in the rat because the post-ganglionic sympathetic nerves which innervate the vas deferens arise from a diffuse hypogastric plexus and do not run in a discrete nerve trunk which could be ligated. However, given that the noradrenergic fibres of the sciatic nerve manifested no alteration in axonal transport of noradrenaline, it is unlikely that such a disorder would be present in other noradrenergic fibres.

The amount of noradrenaline found proximal to the ligature in the constricted sciatic nerves of the control rats is in good agreement with the levels measured in similar experiments by others [2, 14]. The small accumulations distal to the ligature have also been reported and discussed at length by others $[2,3]$.

The finding that noradrenaline accumulation was unaltered by the state of diabetes is in contrast to the results of the only other study in this area. It has been reported that in the genetically diabetic mouse there was a reduction in the amount of noradrenaline accumulated proximal to ligatures applied to the sciatic nerves for $9 \mathrm{~h}$. It may be that the differences in the diabetic state in genetically diabetic mice and chemically diabetic animals [1] confer different effects on nervous tissue. It is also possible that the small and statistically insignificant decrease in the amount of noradrenaline accumulated proximal to the constrictions applied to the sciatic nerves of the 6-month diabetic rats represents an early stage in the development of a chronic disorder. Since the measurement of the amount of noradrenaline accumulated reflects the axonal transport over a $24-h$ period, then a small deficit in such a time span could have a far reaching effect on the supply of material to the nerve terminals over days and weeks. However, it must be stressed that the more well defined defects of axonal transport reported by others in chemically diabetic rats were well established after a period of diabetes much shorter than 6 months [9, 10,15-18]. Thus defects of axonal transport of materials, other than noradrenaline granules, might be more relevant to the search for mechanisms to explain the development of neuropathies.

The reduced response of the vasa deferentia from the chronically diabetic rats to nerve stimulation did not arise as a result of a dysfunction of the smooth muscle cells of the vas. There was no apparent wast- 
ing of the tissue and the responses of the vasa to nonspecific depolarisation by $\mathrm{KCl}$ were similar to those elicited from the vasa of control rats. Thus, it is suggested that a partial denervation of the vasa deferentia had occurred in the chronically diabetic animals. This assertion is supported by the ultrastructural findings.

The hypersensitivity of the vasa deferentia from the chronically diabetic rats to noradrenaline may be attributed to partial denervation of the tissue. A similar hypersensitivity is seen after chemical denervation by 6-hydroxydopamine and is attributed mainly to the absence of removal of the noradrenaline by the nerve terminal [6].

It appears therefore that rats with long-term streptozotocin-induced diabetes develop neuropathy of the noradrenergic supply to the vas deferens. Furthermore, the present findings indicate that this noradrenergic dysfunction is not attributable to disordered axonal transport of noradrenaline. This does not, however, preclude the possibility that damage to nerve terminals and disordered synaptic transmission could arise as a result of impaired axonal transport of other materials.

Acknowledgements. K.R.W.G. was in receipt of a postdoctoral fellowship from the British Diabetic Association. The gift of streptozotocin from the National Institute of Health, Bethesda, Maryland, USA is gratefully acknowledged as is financial support from Imperial Chemical Industries, Pharmaceuticals Division.

\section{References}

1. Coleman DL, Hummel KP (1967) Studies with the mutation, diabetes, in the mouse. Diabetologia 3: 238-248

2. Dahlström A, Häggendal J (1966) Studies on the transport and lifespan of amine storage granules in a peripheral adrenergic neuron system. Acta Physiol Scand 67:278-288

3. Dahlström A (1971) Axoplasmic transport (with particular respect to adrenergic neurons). Philos Trans R Soc London Ser B $261: 325-358$

4. Giachetti A (1979) Axoplasmic transport of noradrenaline in the sciatic nerves of spontaneously diabetic mice. Diabetologia 16: 191-194

5. Giachetti A (1981) Diabetic neuropathies: pathogenetic mechanisms and therapeutic perspectives. Pharmacol Res Commun 13: 101-119
6. Haeusler G (1971) Short and long term effects of 6-hydroxydopamine on peripheral organs. In: Malmfors $T$, Thoenen $H$ (eds) 6-hydroxydopamine and catecholamine neurons. North Holland Publishing Company, Amsterdam, pp 193-204

7. Hjemdahl P, Daleskog M, Kahtan T (1979) Determination of plasma catecholamines by high performance liquid chromatography with electrochemical detection: comparison with a radioenzymatic method. Life Sci 25: 131-138

8. Jakobsen J (1979 Early and preventable changes of peripheral nerve structure and function in insulin-deficiency diabetic rat. $J$ Neurol Neurosurg Psychiatry 42: 509-518

9. Jakobsen J, Sidenius P (1979) Decreased axonal flux of retrogradely transported glycoproteins in early experimental diabetes. J Neurochem 33: 1055-1060

10. Jakobsen J, Sidenius P (1980) Decreased axonal transport of structural proteins in streptozotocin diabetic rats. J Clin Invest 66:292-297

11. Jakobsen J, Sidenius P, Lundbaek K (1980) Nervous system abnormality and nervous disease in diabetes. Acta Endocrinol 94 (Suppl 238): 123-131

12. Káldor A, Gachályi B, Szigeti Á, Juvancz P, Szilágyi A (1977) 'Intrinsic' heart rate in diabetic neuropathy. Int J Clin Pharmacol 15:335-336

13. Lloyd-Mostyn RH, Watkins PJ (1976) Total cardiac denervation in diabetic autonomic neuropathy. Diabetes 25:748-751

14. McLean WG, Keen P (1972) Local synthesis and breakdown of noradrenaline in constricted rat sciatic nerves. Eur $\mathbf{J}$ Pharmacol $18: 74-78$

15. McLean WG, Meiri KF (1980) Rapid axonal transport of protein in rat sciatic motor nerves during early experimental diabetes. J Physiol 301 : 43-44P

16. Schmidt RE, Matschinsky FM, Godfrey DA, Williams AD, McDougal DB (1975) Fast and slow axoplasmic flow in sciatic nerve of diabetic rats. Diabetes 24: 1081-1085

17. Sidenius P, Jakobsen J (1979) Axonal transport in early experimental diabetes. Brain Res 173:315-330

18. Sidenius P, Jakobsen J (1980) Impaired retrograde axonal transport from a nerve crush in streptozotocin diabetic rats. Diabetologia 19:222-228

19. Tomlinson DR (1979) On the mechanism of pancuronium-induced supersensitivity to noradrenaline in rat smooth muscle. Br J Pharmacol 65: 473-478

20. Tranzer JP, Richards JG (1976) Ultrastructural cytochemistry of biogenic amines in nervous tissue: methodologic improvements. J Histochem Cytochem 24: 1178-1193

Received: 18 May 1981

and in revised form: 24 August 1981

Dr. D. R. Tomlinson

Department of Physiology and Pharmacology

Medical School

Queen's Medical Centre

Nottingham NG7 2UH, UK 